\title{
Long Non-Coding RNA Myosin Light Chain Kinase Antisense I Plays an Oncogenic Role in Gallbladder Carcinoma by Promoting Chemoresistance and Proliferation
}

\author{
Yongliang $\mathrm{Li}^{1}{ }^{1} *$ \\ Mi Tian ${ }^{2} *$ \\ Dongqing Zhang' \\ Yifei Zhuang' \\ Zhimin $\mathrm{Li}^{\prime}$ \\ Shenqi Xie' \\ Keyu Sun'
}

'Department of Emergency, Minhang Hospital, Fudan University, Shanghai, 200040, People's Republic of China;

${ }^{2}$ Department of Intensive Care Unit, Huashan Hospital, Fudan University, Shanghai, 200040, People's Republic of China

*These authors contributed equally to this work
Correspondence: Keyu Sun Department of Emergency, Minhang Hospital, Fudan University, Shanghai, 200040, People's Republic of China $\mathrm{Tel}+86$ |893053686I

Email hoceima@126.com
Background: Long non-coding RNAs (lncRNAs) have been reported to play critical roles in human tumours, including gallbladder carcinoma (GBC). However, their biological functions and molecular mechanisms in tumorigenesis and progression remain largely unknown. Methods: Quantitative polymerase chain reaction (qPCR) was used to verify the expression of lncRNA myosin light chain kinase antisense RNA 1 (MYLK-AS1) in 120 pairs of GBC tissues and paired adjacent non-tumour tissues, as well as in six different GBC cell lines (NOZ, EH-GB1, OCUG-1, GBC-SD, SGC-996 and QBC-939). Cell counting kit 8 was applied to explore cell proliferation and drug sensitivity assays. The target miRNAs (miR) of MYLK-AS1 and downstream target genes were predicted using Starbase 3.0 software and confirmed by double luciferase reporting test. The expression of proteins was assessed using Western blot assay.

Results: Here, we demonstrated that MYLK-AS1 was significantly upregulated and correlated with a poor prognosis and poor clinical characteristics in GBC. Furthermore, the forced expression of MYLK-AS1 significantly promoted GBC cell proliferation and resistance to gemcitabine in vitro. Mechanistically, MYLK-AS1 functioned as an efficient miR-217 sponge, thereby releasing the inhibition of enhancer of zeste 2 polycomb repressive complex 2 (EZH2) subunit expression. MYLK-AS1 promoted GBC cell proliferation and resistance to gemcitabine by upregulating EZH2 expression, and EZH2 was confirmed as a direct target of miR-217.

Discussion: Our results confirmed that the chemoresistant driver MYLK-AS1 might be a promising candidate as a therapeutic target for the treatment of advanced GBC.

Keywords: chemoresistance, GBC, lncRNA, proliferation

\section{Introduction}

Gallbladder carcinoma (GBC) has a poor prognosis due to local recurrence, metastasis, and resistance to targeted therapies. ${ }^{1}$ Gemcitabine is widely used as the firstline treatment in locally advanced and metastatic GBC. ${ }^{2}$ However, patient prognosis is usually limited by natural and acquired chemotherapy resistance. Thus, it is critical to identify potential chemotherapy resistance molecular mechanisms to restore GBC cell sensitivity to gemcitabine.

Long non-coding RNAs (lncRNAs) are a class of non-coding RNAs characterized as transcripts of more than 200 nucleotides that do not have protein encoding 
functions. ${ }^{3}$ Recently, an increasing number of studies have reported that the dysregulation of IncRNAs participates in regulating the tumorigenesis, progression, and chemoresistance of human tumours. ${ }^{4}$ For example, lncRNA small Cajal body-specific RNA 2 (SCARNA2) promotes chemotherapy resistance by competitively binding to miR$342-3 p$ to facilitate epidermal growth factor receptor (EGFR) and B-cell lymphoma 2 (BCL2) expression in colorectal cancer cells. ${ }^{5}$ Recently, several studies have reported that lncRNA myosin light chain kinase antisense RNA 1 (MYLK-AS1) expression is increased in several malignant tumours and functions as an oncogene in several types of cancer, including gastric cancer, nephroblastoma, and hepatocellular carcinoma. ${ }^{6-8}$ However, the clinical relevance or biological functions of MYLK-AS1 in GBC are currently unknown.

The forced expression of enhancer of zeste homologue 2 (EZH2) has been observed in numerous tumours, including GBC. ${ }^{9-12}$ EZH2 plays a critical role in the physiological and pathological processes of cells, including cancer cell proliferation, invasion, metastasis, angiogenesis, and chemotherapy resistance. ${ }^{13}$ In this study, we found that the expression of MYLK-AS1 was upregulated in GBC tissues compared with adjacent non-tumour tissues. The forced expression of MYLK-AS1 predicted a worse prognosis in patients with GBC. MYLK-AS1 promoted proliferation and conferred resistance to gemcitabine by upregulating the expression of EZH2 by sponging miR217 in GBC cells. Thus, our results revealed a novel role for the MYLK-AS1/miR-217/EZH2 axis in regulating the proliferation and chemotherapy resistance of GBC cells.

\section{Materials and Methods}

\section{Cell Culture and Transfection}

Human GBC cell lines (NOZ, EH-GB1, OCUG-1, GBCSD, SGC-996 and QBC-939), an immortalized human non-tumorigenic biliary epithelial cell line (H69) and the HEK-293T cell line were obtained from the Cell Bank of the Chinese Academy of Sciences (Shanghai, China) and cultured in optimal culture medium containing $10 \%$ foetal bovine serum (FBS, Gibco, USA). All of the cells were cultured in a humidified incubator containing $5 \% \mathrm{CO}_{2}$ at $37{ }^{\circ} \mathrm{C}$. The microRNA (miRNA) mimics, miRNA inhibitor, and negative control utilized for transfection were purchased from GenePharma (Shanghai, China). MYLKAS1 overexpression and short hairpin RNA adenoviruses were purchased from Genomeditech (Shanghai, China), and the transfection procedure was performed in accordance with the manufacturer's protocol. The EZH2 plasmid was purchased from GenePharma (Shanghai, China) and transfected via Lipofectamine 2000 Reagent (Invitrogen) in accordance with the manufacturer's protocol.

\section{Human Tissue Samples}

GBC tissues and adjacent non-tumour tissues were obtained from patients who were diagnosed and underwent surgery in Minhang Hospital Affiliated to Fudan University and stored in liquid nitrogen and formalin. This study was approved by the Joint Ethics Committee of Minhang Hospital Affiliated to Fudan University, and written informed consent was obtained from all the patients. Experiments were conducted in accordance with the Declaration of Helsinki.

\section{RNA Isolation and Reverse Transcription-Quantitative Polymerase Chain Reaction (RT-qPCR)}

Total RNA was extracted from tissues and cells with TRIzol reagent (Invitrogen) according to the manufacturer's protocol. To detect MYLK-AS1 and EZH2 mRNA expression, the PrimeScript RT Reagent Kit (TaKaRa) and SYBR Premix Ex Taq II Kit (TaKaRa) were used according to the manufacturer's protocol. GAPDH was used as an internal control. To detect miR217 expression, the Mir- $\mathrm{X}^{\mathrm{TM}}$ miRNA First Strand Synthesis Kit and Mir-X ${ }^{\mathrm{TM}}$ miRNA RT-qPCR SYBR ${ }^{\circledR}$ Kit were used according to the manufacturer's protocol. U6 was used as an internal control. The primers used, synthesized by BGI Co. Ltd., are listed as follows: MYLK-AS1-F: CCTAGAATCCAGTGTCGTCC; MYLKAS1-R: CTTCGACGACGAGGCATTCG; EZH2-F: ATC CTGACTTCTGTGAGCTC; EZH2-R: TCATCTCCCAT ATAAGGAAT-G; GAPDH-F: GTACGTCGTGGAGTCC ACTG; and GAPDH-R: CTTCCACGATACC-AAAG TTG. The relative expression was analysed using the $2^{-\Delta \Delta \mathrm{Ct}}$ method.

\section{Proliferation and Drug Sensitivity Assays}

Cell proliferation and drug sensitivity were carried out using the Cell Counting Kit-8 (CCK-8) (Beyotime Biotechnology) according to a previously published reference. ${ }^{5}$ For the proliferation assay, 500 tumour cells were cultured in 96-well plates for $24,48,72,96$, and 
$120 \mathrm{~h}$. Then, the CCK-8 solution (Dojindo) was added to the cells and cultured in a humidified incubator containing $5 \% \mathrm{CO}_{2}$ at $37{ }^{\circ} \mathrm{C}$. After $2 \mathrm{~h}$, the optical density (OD) was measured at $450 \mathrm{~nm}$. For the drug sensitivity assay, 2000 tumour cells were cultured in 96-well plates for $24 \mathrm{~h}$. Then, the cultured medium containing different concentrations of the chemotherapy agent was added to the cells, which were then cultured in a humidified incubator containing $5 \% \mathrm{CO}_{2}$ at $37^{\circ} \mathrm{C}$. After $2 \mathrm{~h}$, the optical density (OD) was measured at $450 \mathrm{~nm}$, and the IC50 was calculated.

\section{Prediction of MYLK-ASI and EZH2 mRNA Binding to miR-2I7 and Luciferase Reporter Assays}

StarBase V3.0 was used to predict that MiR-217 could bind to MYLK-AS1 and EZH2 mRNA. Luciferase reporter assays were performed as described in a previously published reference. ${ }^{14}$ HEK-293T cells were seeded in 96-well plates. After $24 \mathrm{~h}$, cells were cotransfected with the miR-217 mimics/negative control and the corresponding luciferase reporter vectors $(\mathrm{wt} / \mathrm{mu}$ MYLK-AS1 and EZH2), after which a dual luciferase reporter assay system (Promega, Madison, WI, USA) was used.

\section{Western Blotting Analysis}

Samples were rifted in RIPA lysis buffer plus PMSF with low temperature, and BCA assay kit (Beyotime Biotechnology) examined total protein concentration. Protein samples $(20 \mu \mathrm{g})$ were separated via sodium dodecyl sulfate-polyacrylamide gel electrophoresis and transferred onto polyvinylidene difluoride (PVDF) membranes and then were incubated with prepared antibodies. Finally, this membrane was visualized by enhanced chemiluminescence (ThermoFisher). Antibodies against GAPDH and EZH2 were purchased from Abcam.
A

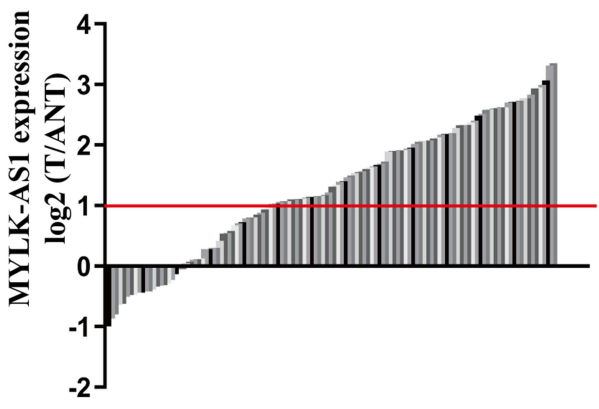

D

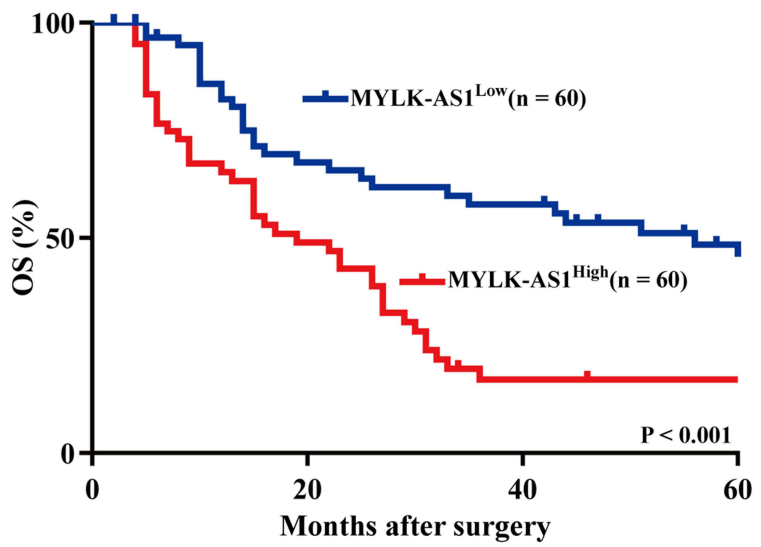

B

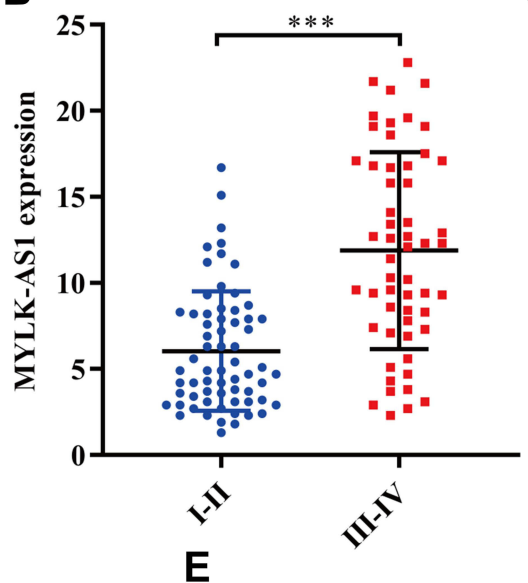

C

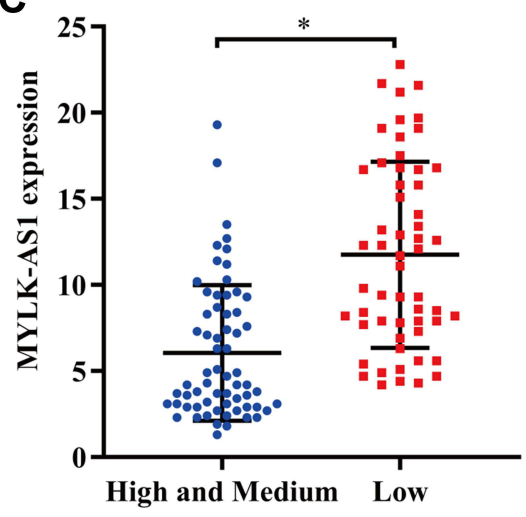

Figure I Overexpression of MYLK-ASI predicts a poor prognosis in GBC patients. (A) The expression levels of MYLK-ASI in GBC tissues and corresponding adjacent non-cancerous tissues were detected by RT-qPCR. (B and C) A total of I20 GBC patients were divided into well or moderate differentiation and low differentiation/I-II stage and III-IV stage groups. The diagram shows MYLK-ASI expression in each group. *P $<0.05$; ***P $<0.00 I$. (D and $\mathbf{E})$ Kaplan-Meier analysis of overall survival and PFS in GBC patients with low and high MYLK-ASI levels. 


\section{Construction of Tissue Microarrays} (TMAs) and Immunohistochemistry (IHC) Staining

TMA and IHC staining analyses of EZH2 were performed as previously described. ${ }^{5}$ In brief, TMA was prepared using classical methods, and EZH2 expression was detected using an immunoperoxidase method. According to the intensity and total area of the staining, EZH2 expression levels were classified as a reference.

\section{Statistical Analysis}

All statistical analyses were performed using SPSS 18.0 software (Chicago, IL, USA) and GraphPad Prism 7 as a reference. ${ }^{15} \mathrm{P}<0.05$ was considered statistically significant. These experiments were carried out independently at least three times.

\section{Results}

\section{MYLK-ASI Overexpression Indicated the} Poor Prognosis of GBC

To investigate the possible biological functions of MYLKAS1 in the progression of GBC, we first detected the expression of MYLK-AS1 in 120 pairs of GBC tissues and paired adjacent non-tumour tissues. As shown by RT-qPCR results, MYLK-AS1 expression was higher in GBC tumour tissues than in paired adjacent non-tumour tissues (77/120) (Figure 1A). Next, we investigated the relationship between MYLK-AS1 expression and the clinicopathological characteristics of $120 \mathrm{GBC}$ patients, as listed in Table 1 . The results demonstrated that GBC patients with MYLK-AS $1^{\text {high }}$ had an advanced TNM stage $(\mathrm{P}=0.001)$ (Figure $1 \mathrm{~B})$ and low differentiation $(\mathrm{P}=0.006)$ (Figure $1 \mathrm{C})$. We then analysed the prognostic implications of MYLK-AS1 expression. Importantly, we found that patients with a high level of MYLK-AS1 expression had a significantly worse prognosis than those with MYLK-AS1 $1^{\text {low }}$ expression (Figure $1 \mathrm{D}$ and E). Multivariate analysis identified that forced MYLK-AS1 expression was an independent prognostic predictor for GBC patients (Tables 2 and 3). These results indicated that MYLK-AS1 likely participates in the progression of GBC.

\section{MYLK-ASI Promoted Proliferation and Chemotherapy Resistance}

Next, we explored the biological functions of MYLK-AS1 in GBC. Because GBC-SD and NOZ cells have a relatively high expression of MYLK-AS1, whereas EH-GB1 and
Table I The Correlation Between Clinicopathological Characteristics and MYLK-ASI Expression

\begin{tabular}{|c|c|c|c|}
\hline \multirow[t]{2}{*}{ Clinicopathological Characteristics } & \multicolumn{2}{|c|}{$\begin{array}{l}\text { MYLK-ASI } \\
\text { Expression }\end{array}$} & \multirow[t]{2}{*}{$\boldsymbol{P}$} \\
\hline & Low & High & \\
\hline \multicolumn{4}{|l|}{ Age } \\
\hline$\leq 60$ & 23 & 28 & 0.767 \\
\hline$>60$ & 33 & 36 & \\
\hline \multicolumn{4}{|l|}{ Sex } \\
\hline Male & 42 & 47 & 0.845 \\
\hline Female & 14 & 17 & \\
\hline \multicolumn{4}{|l|}{ Alcohol consumption } \\
\hline Ever and current & 31 & 42 & 0.250 \\
\hline Never & 25 & 22 & \\
\hline \multicolumn{4}{|l|}{ Smoking status } \\
\hline Ever and current & 23 & 32 & 0.327 \\
\hline Never & 33 & 32 & \\
\hline \multicolumn{4}{|l|}{ Tumor size } \\
\hline$<3 \mathrm{~cm}$ & 30 & 31 & 0.574 \\
\hline$\geq 3 \mathrm{~cm}$ & 26 & 33 & \\
\hline \multicolumn{4}{|l|}{ Differentiation status } \\
\hline Well or Moderate & 37 & 26 & 0.005 \\
\hline Poor & 19 & 38 & \\
\hline \multicolumn{4}{|l|}{ TNM stage } \\
\hline I-II & 39 & 26 & 0.001 \\
\hline III-IV & 17 & 38 & \\
\hline
\end{tabular}

SGC996 cells have a relatively low expression of MYLKAS1 (Figure 2A), we silenced the expression of endogenous MYLK-AS1 in GBC-SD and NOZ cells using shRNA and overexpressed MYLK-AS1 in EH-GB1 and SGC996 cells using an expression vector containing full-length human MYLK-AS1. RT-qPCR analysis was used to detect the transfection efficiency (Figure $2 \mathrm{~B}$ and $\mathrm{C}$ ). The CCK-8 assay demonstrated that the knockdown of MYLK-AS1 inhibited the proliferation of GBC-SD and NOZ cells and increased the chemotherapy sensitivity of GBC-SD and NOZ cell to gemcitabine (Figure 2D and E). Conversely, the upregulation of MYLK-AS1 promoted the proliferation of EH-GB1 and SGC996 cells and induced EH-GB1 and SGC996 cell resistance to gemcitabine (Figure 2F and G).

\section{MiR-2 I7 Was a Direct Target of MYLK-ASI}

Recently, accumulating evidence confirmed that lncRNAs mainly function as competing endogenous 
Table 2 Univariate and Multivariate Analyses of Factors Associated with Overall Survival

\begin{tabular}{|c|c|c|c|c|}
\hline \multirow[t]{3}{*}{ Clinicopathological Characteristics } & \multicolumn{4}{|c|}{ OS } \\
\hline & \multirow{2}{*}{$\begin{array}{c}\text { Univariate } \\
\mathbf{P}\end{array}$} & \multicolumn{3}{|c|}{ Multivariate } \\
\hline & & HR & $95 \% \mathrm{Cl}$ & $P$ value \\
\hline Sex (female vs male) & 0.317 & & & NA \\
\hline Age (years) $(\leq 60$ vs $>60)$ & 0.822 & & & NA \\
\hline Alcohol consumption (Ever and current vs never) & 0.671 & & & NA \\
\hline Smoking status (Ever and current vs never) & 0.073 & & & NA \\
\hline Tumor size (diameter, $\mathrm{cm}$ ) (>3 vs $\leq 3$ ) & 0.064 & & & NA \\
\hline Differentiation status (Well or Moderate vs Poor) & 0.024 & & & NS \\
\hline TNM (III/IV vs I/II) & 0.016 & & & NS \\
\hline MYLK-ASI expression (high vs low) & 0.002 & 1.322 & $1.107-2.092$ & 0.017 \\
\hline
\end{tabular}

Abbreviations: NA, not adopted; NS, not significant; $95 \% \mathrm{Cl}, 95 \%$ confidence interval; HR, hazard ratio; Cox proportional hazards regression model.

Table 3 Univariate and Multivariate Analyses of Factors Associated with Cumulative Recurrence

\begin{tabular}{|c|c|c|c|c|}
\hline \multirow[t]{3}{*}{ Clinicopathological Characteristics } & \multicolumn{4}{|c|}{ Cumulative Recurrence } \\
\hline & \multirow{2}{*}{$\frac{\text { Univariate }}{P}$} & \multicolumn{3}{|c|}{ Multivariate } \\
\hline & & HR & $95 \% \mathrm{Cl}$ & $P$ value \\
\hline Sex (female vs male) & 0.146 & & & NA \\
\hline Age (years) $(\leq 60$ vs $>60)$ & 0.276 & & & NA \\
\hline Alcohol consumption (Ever and current vs never) & 0.733 & & & NA \\
\hline Smoking status (Ever and current vs never) & 0.463 & & & NA \\
\hline Tumor size (diameter, $\mathrm{cm})(>3$ vs $\leq 3$ ) & 0.084 & & & NA \\
\hline Differentiation status (Well or Moderate vs Poor) & 0.031 & & & NA \\
\hline TNM (III/IV vs I/II) & 0.007 & 1.034 & $0.811-1.972$ & 0.042 \\
\hline MYLK-ASI expression (high vs low) & 0.004 & 1.472 & $1.085-2.723$ & 0.025 \\
\hline
\end{tabular}

Abbreviations: NA, not adopted; NS, not significant; $95 \% \mathrm{Cl}$, 95\% confidence interval; HR, hazard ratio; Cox proportional hazards regression model.

RNAs (ceRNAs) by sponging miRNAs. Thus, StarBase v3.0 was used to analyse the potential miRNAs that bound to MYLK-AS1, and the results showed that 28 miRNAs might bind to MYLK-AS1, including miR-217, a tumour suppressor miRNA. Therefore, we predicted miR-217 to be a potential target of MYLK-AS1 via StarBase V3.0 (Figure 3A). To further verify whether MYLK-AS1 could bind to miR-217 and act as a ceRNA, we constructed luciferase reporter vectors of MYLK-AS1 containing wild-type (wt) or mutated (mu) miR-217 binding sites. Luciferase reporter assays revealed that cotransfection of miR-217 with wt MYLKAS1 significantly inhibited luciferase activity compared with cotransfection of miR-217 with mu MYLK-AS1 (Figure 3B). Furthermore, in MYLK-AS1overexpressing GBC cells, miR-217 expression was repressed, while miR-217 expression was upregulated in MYLK-AS1 knockdown GBC cells (Figure 3C and
D). These results indicated that MYLK-AS1 sponges miR-217 in GBC cells.

\section{Inhibition of miR-2 17 Restrained the Proliferation and Chemotherapy Resistance of GBC Cells}

To further confirm the interaction of miR-217 with MYLK-AS1, we assessed its influence on the proliferation and chemotherapy resistance of GBC cells. A miR217-specific inhibitor was synthesized and used to downregulate the expression of miR-217 in GBC-SDMYLK-AS1 shRNA and NOZ-MYLK-AS1 shRNA cells (Figure 4A). Cell proliferation assays showed that downregulated miR-217 had inhibitory effects on cell proliferation capabilities in GBC-SD-MYLK-AS1 shRNA and NOZ-MYLK-AS1 shRNA cells (Figure 4B and $\mathrm{C}$ ). In addition, a drug sensitivity assay 
A

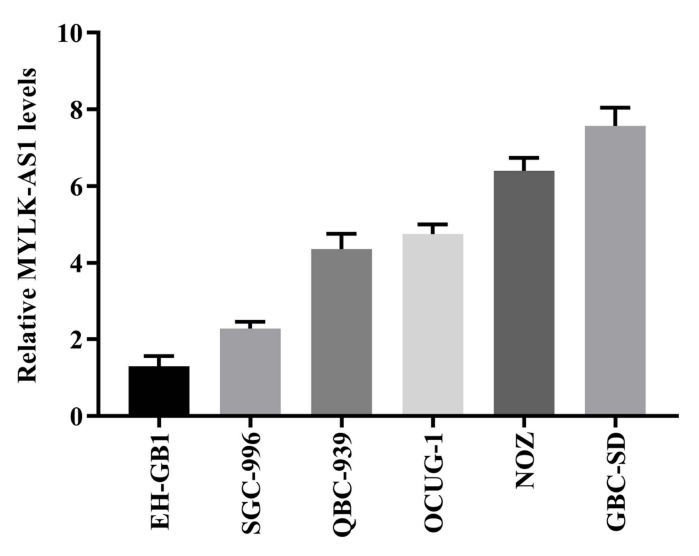

D
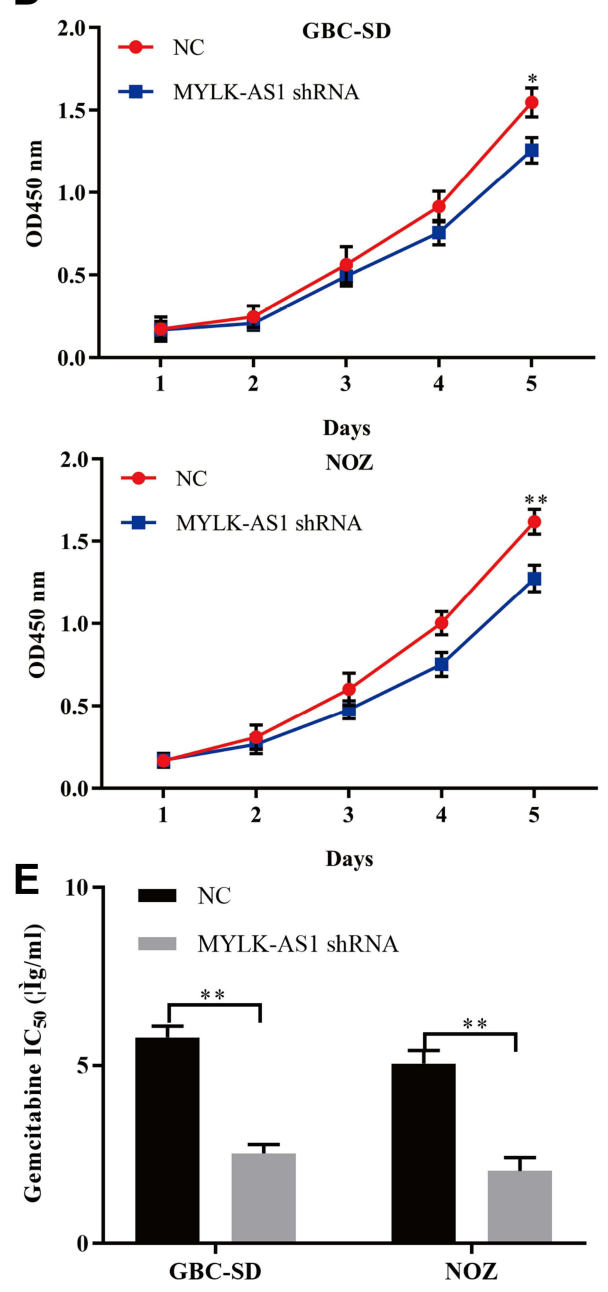

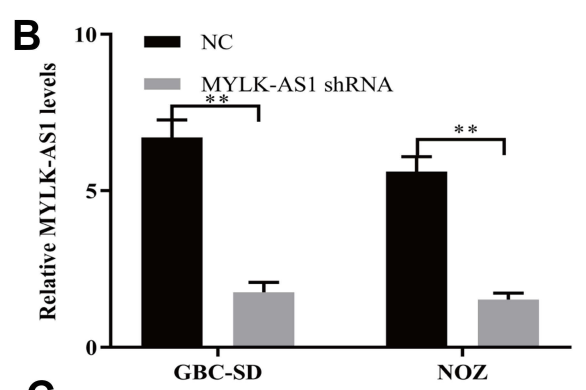

$\mathrm{C}_{10} \underset{\mathrm{MBC}-\mathrm{SD}}{\mathrm{Mock}}$
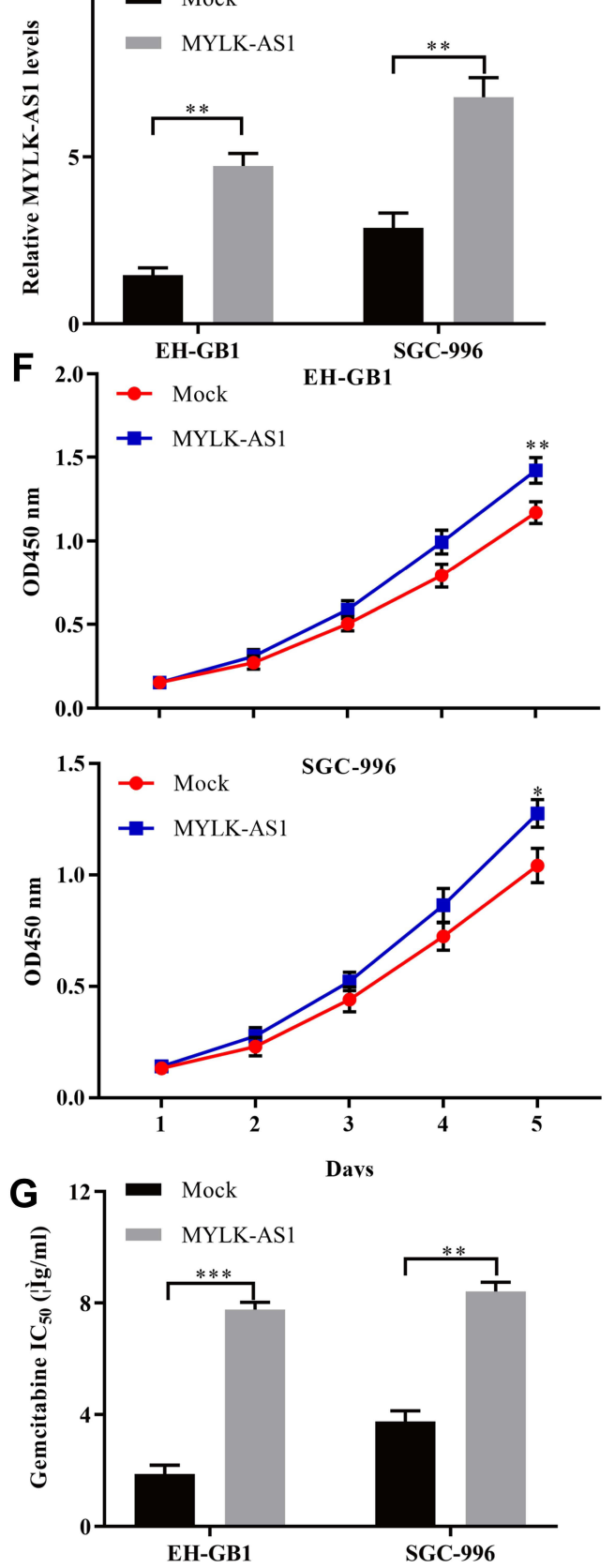

Figure 2 Forced MYLK-ASI expression promotes GBC cell resistance to gemcitabine in vitro. (A) MYLK-ASI expression in six GBC cell lines was examined using RT-qPCR analysis. (B and C) MYLK-ASI expression in GBC cells was modified by shRNA interference and cDNA transfection. (D-G) Cell proliferation and drug sensitivity were measured using CCK-8 assays. The data are represented as the mean $\pm \mathrm{SD}, \mathrm{n}=3$. $* \mathrm{P}<0.05 ; * * \mathrm{P}<0.01 ; * * * \mathrm{P}<0.001$. 
A

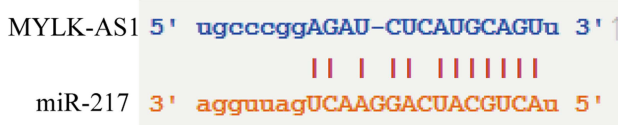

C

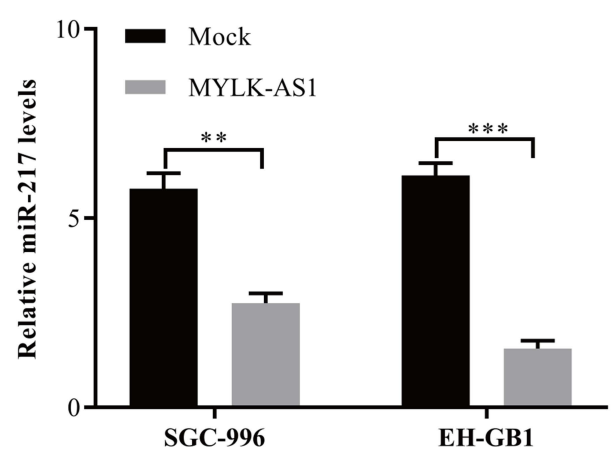

B
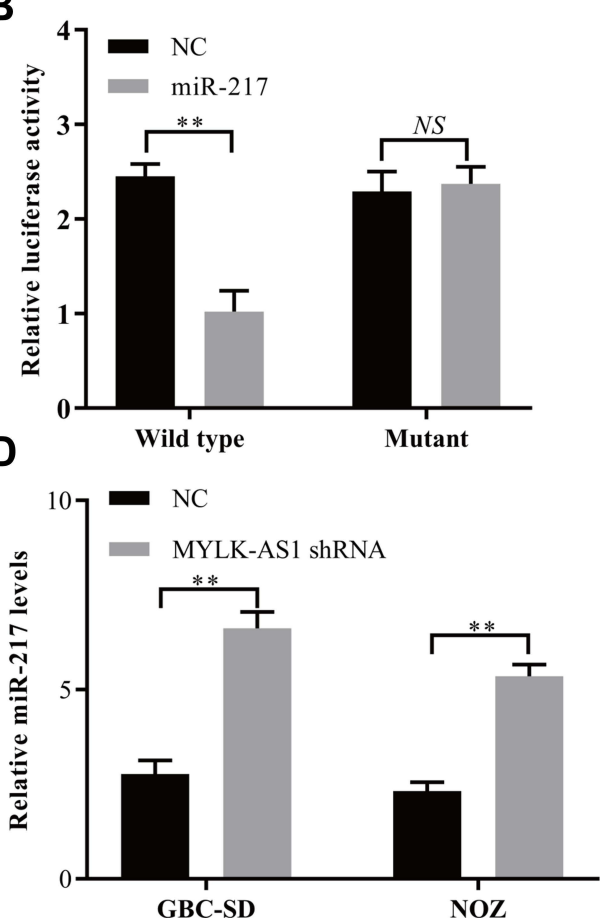

Figure 3 MYLK-ASI functions as a ceRNA for miR-2I7 in GBC cells. (A) Target sequences in MYLK-ASI predicted to bind to miR-2I7. (B) Wild-type or mutant MYLKASI was transfected into HEK-293T cells with synthetic miR-2I7 or negative control. Luciferase activity was detected $48 \mathrm{~h}$ after transfection. (C and D) miR-2 I7 expression after upregulation or knockdown of MYLK-ASI expression was detected in GBC cells by using RT-qPCR. The data are represented as the mean \pm SD, $n=3$. **P $<0.0$ I; $* * * \mathrm{P}<0.001$.

demonstrated that inhibition of miR-217 significantly decreased the sensitivity of GBC-SD-MYLK-AS1 shRNA and NOZ-MYLK-AS1 shRNA cells to gemcitabine (Figure 4D). Taken together, our results suggested that MYLK-AS1 can promote the proliferation and chemotherapy resistance of $\mathrm{GBC}$ cells by targeting miR-217.

\section{EZH2 Was Confirmed as a Direct Target} Gene of miR-217

To further investigate the role of MYLK-AS1 in GBC, we predicted miR-217 targets using StarBase v3.0. As shown in Figure 5A, the 3'-UTR of EZH2 mRNA contains a putative binding site of miR-217. A luciferase reporter assay was also used to validate the direct interaction between EZH2 and miR-217 (Figure 5B). Furthermore, the mRNA levels of EZH2 in EH-GB1 and SGC996 cells were reduced after transfection with miR-217 mimics (Figure 5C). Conversely, the mRNA levels of EZH2 in GBC-SD and NOZ cells were increased after transfection with the inhibitor (Figure 5D).

\section{MYLK-ASI Regulated the miR-2I7/ EZH2-Mediated Signalling Pathway in GBC}

The upregulation of EZH2 is closely related to tumour progression, including GBC. ${ }^{16-19}$ Here, we posited that MYLK-AS1 may promote GBC cell resistance to chemotherapy agents by regulating the miR-217/EZH2mediated signalling pathway. To test our hypothesis, we examined the expression of miR-217 and EZH2 in tissues from $120 \mathrm{GBC}$ tissue samples. The results showed that there was a negative correlation between miR-217 and MYLK-AS1 in GBC tissues (Figure 6A). Furthermore, positive correlations between MYLK-AS1 and EZH2 were observed in GBC tissues (Figure 6B). To further investigate the results that MYLK-AS1 promoted GBC cell proliferation and chemotherapy resistance through regulation of the miR-217/EZH2 axis, we investigated the expression of MYLK-AS1 on EZH2 via Western blotting and RT-qPCR. The results showed that forced MYLK-AS1 expression led to increased protein and mRNA expression levels of EZH2, while decreased MYLK-AS1 downregulated EZH2 expression in GBC 

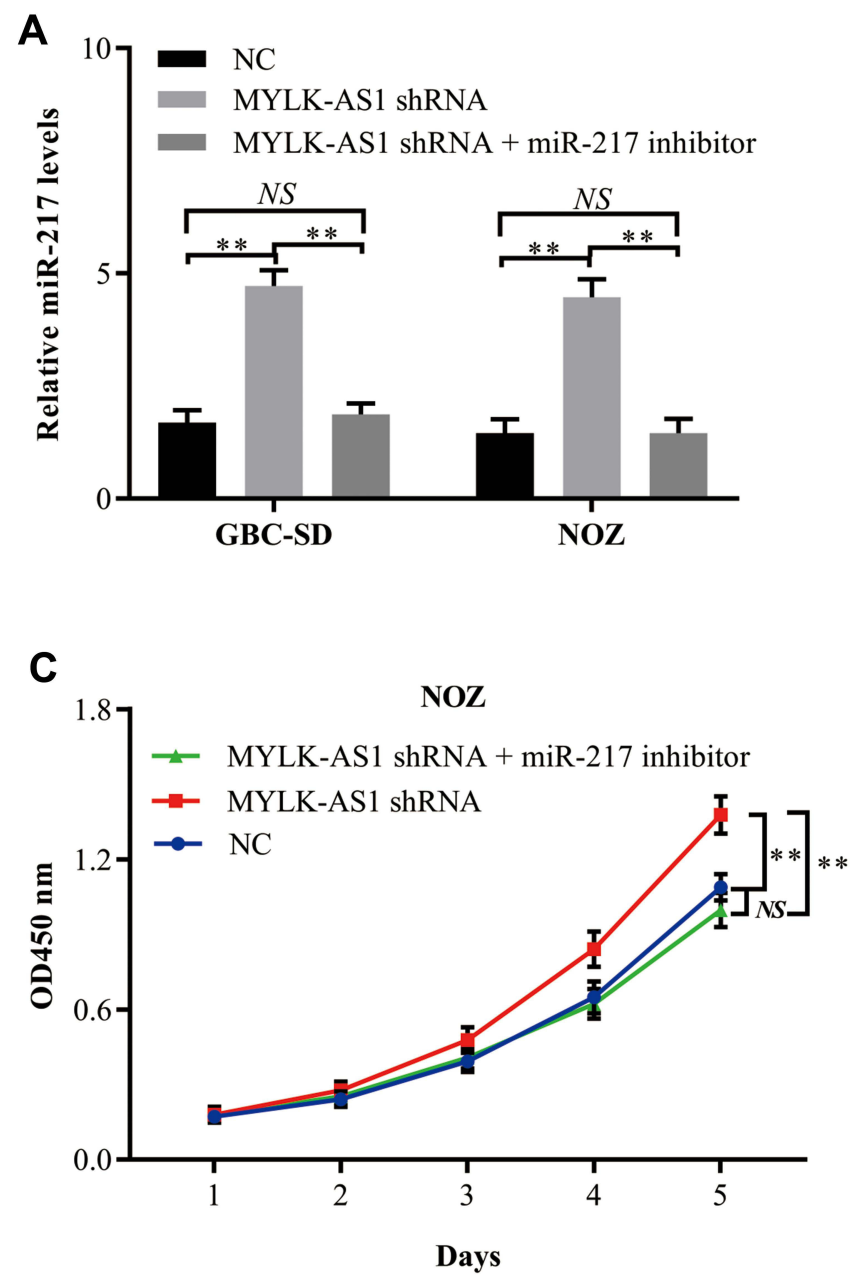

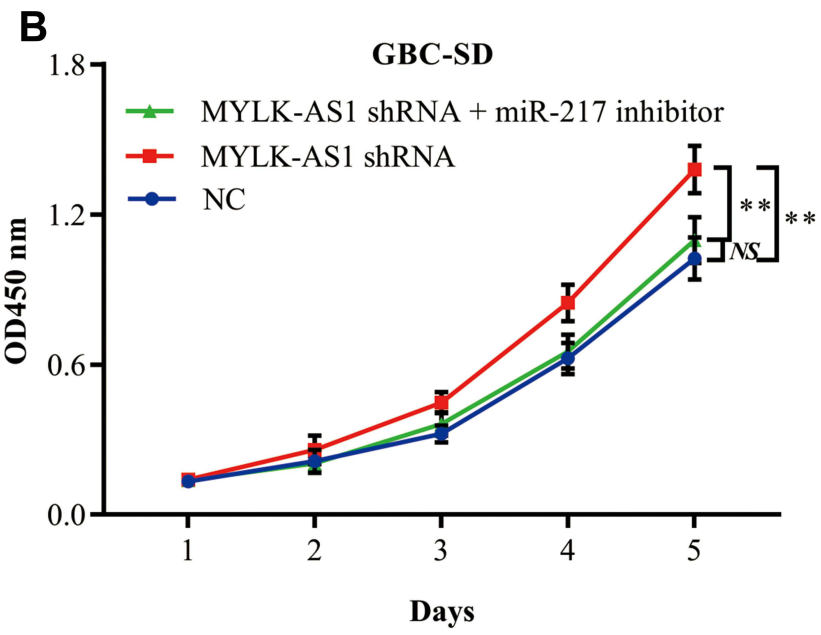

D

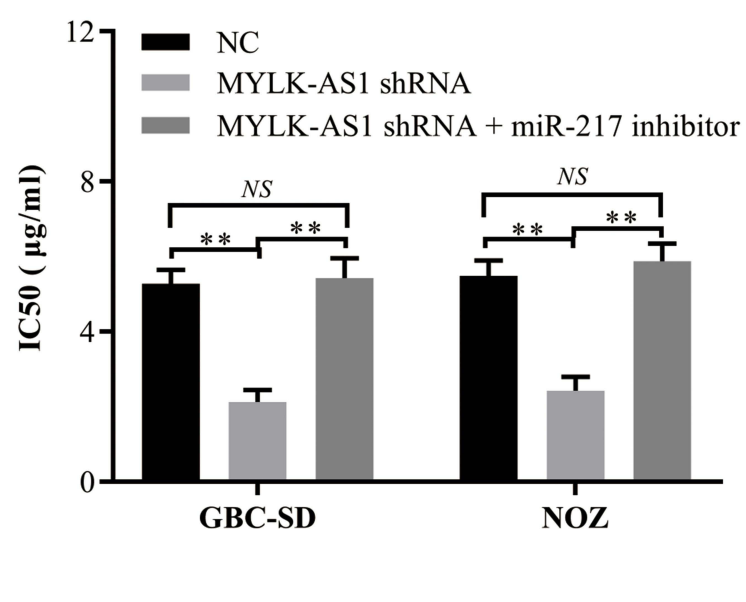

Figure 4 Silencing of miR-2I7 impairs the cell proliferation and chemoresistance inhibition of GBC cells with decreased MYLK-ASI expression. (A) miR-2I7 expression in GBC cells was modified by shRNA interference. (B and C) Cell proliferation in GBC cells with reduced expression of miR-I9I was assessed by a CCK-8 assay. (D) Drug sensitivity in GBC cells with reduced expression of miR-2I7 was evaluated via a CCK-8 assay. The data are represented as the mean \pm SD, $n=3$. $* * P<0.01$; NS, not significant.

cells (Figure 6C and D). To further verify our hypothesis, we established EZH2-overexpressing EH-GB1 and SGC996 cells (EH-GB1-E and SGC996-E cells) through transfection with the EZH2 plasmid (Figure 6E). Proliferation and drug sensitivity assays showed that the increased $\mathrm{EZH} 2$ expression promoted the proliferation and chemoresistance of EH-GB1 and SGC996 cells (Figure 6F-H).

\section{Discussion}

In this study, we reported that MYLK-AS1 upregulated the expression of EZH2 by acting as a sponge for miR217, as shown in molecular and functional assays, and thus promoted the proliferation and chemoresistance of GBC. This study is the first to report that the expression of $\mathrm{EZH} 2$ in GBC is regulated by MYLK-AS1 and the first to show the molecular mechanism of MYLK-AS1 in GBC.

Recently, several studies reported that miR-217 functions as a tumour suppressor in multiple cancers by targeting oncogenes. $^{20-24}$ For example, overexpression of miR-217 can suppress colony formation and the cell invasion capacity by targeting Rho-associated protein kinase 1 (ROCK1) in cervical cancer cells. ${ }^{25}$ In colorectal cancer cell lines, upregulation of miR-217-5p expression induces cancer cell apoptosis by inhibiting PRKCI, BAG3, ITGAV and MAPK1. ${ }^{24}$ Importantly, inhibiting miR-217 expression enhances chemoresistance in several types of cancer. ${ }^{26-28}$ However, the role of miR-217 in drug resistance in GBC remains unknown. To explore the underlying molecular mechanisms of MYLK-AS1, we validated miR-217 as a direct target of MYLK-AS1 in GBC cells. Dual-luciferase reporter and RIP 

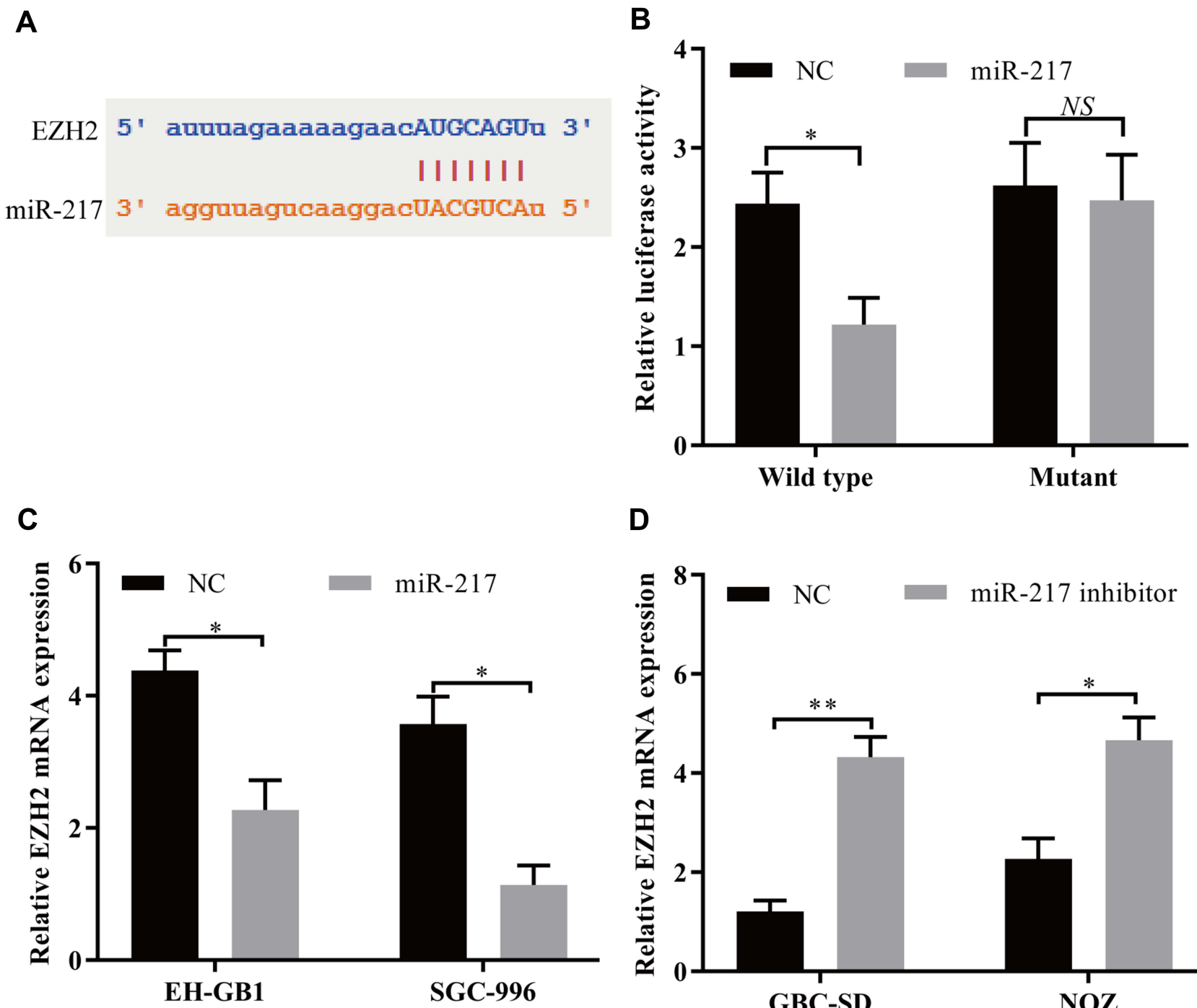

D

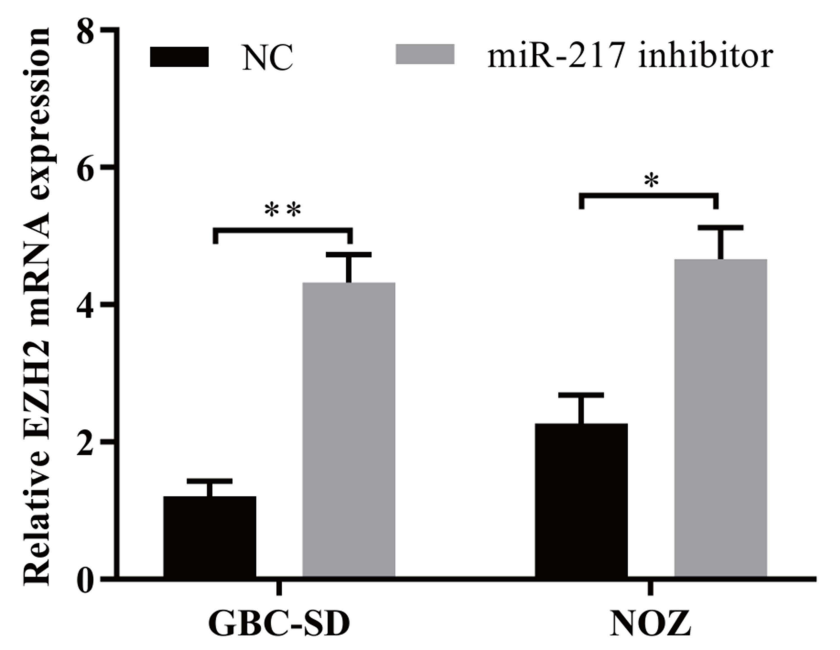

Figure 5 EZH2 mRNA acts as a target for miR-2I7 in GBC cells. (A) Target sequences in EZH2 mRNA predicted to bind to miR-2।7. (B) Wild-type or mutant EZH2 3'UTR was transfected into HEK-293T cells with synthetic miR-2I7 or negative control. Luciferase activity was detected $48 \mathrm{~h}$ after transfection. (C and D) EZH2 mRNA expression after upregulation or knockdown of miR-2I7 expression was detected in GBC cells by using RT-qPCR. The data are represented as the mean \pm SD, $\mathrm{n}=3$. $* \mathrm{P}<$ $0.05 ; * * \mathrm{P}<0.01$.

assays demonstrated the direct interaction between MYLKAS1 and miR-217.

EZH2 is an important component of polycombrepressive complex 2 (PRC2) and participates in suppressing gene expression by catalysing histone 3 lysine 27 trimethylation. ${ }^{29}$ Recently, EZH2 has been reported to be an oncogene involved in the progression of various cancers, including GBC. Here, our results showed that aberrant MYLK-AS1/miR-217 may be associated with the regulation of EZH2, thus regulating chemotherapy resistance in GBC. Interestingly, EZH2 was predicted and validated as a target of miR-217 using StarBase 3.0 and luciferase reporter assays.
The expression of EZH2 in GBC cells treated with MYLK-AS1 was dramatically increased, and the knockdown of MYLK-AS1 significantly decreased the level of EZH2, revealing the regulatory role of MYLKAS1 on EZH2 expression. Importantly, the dysregulation of EZH2 has been reported to be involved in chemotherapy resistance. For example, inhibition of EZH2 by inhibitor or miRNA mimics can reverse docetaxel resistance by suppressing cancer stem cells in prostate cancer. ${ }^{30}$ In breast cancer, EZH2 confers tamoxifen resistance by inhibiting the expression of the oestrogen receptor $\alpha(E R \alpha)$ cofactor GREB $1 .{ }^{31}$ Overall, our results and previous studies suggest 

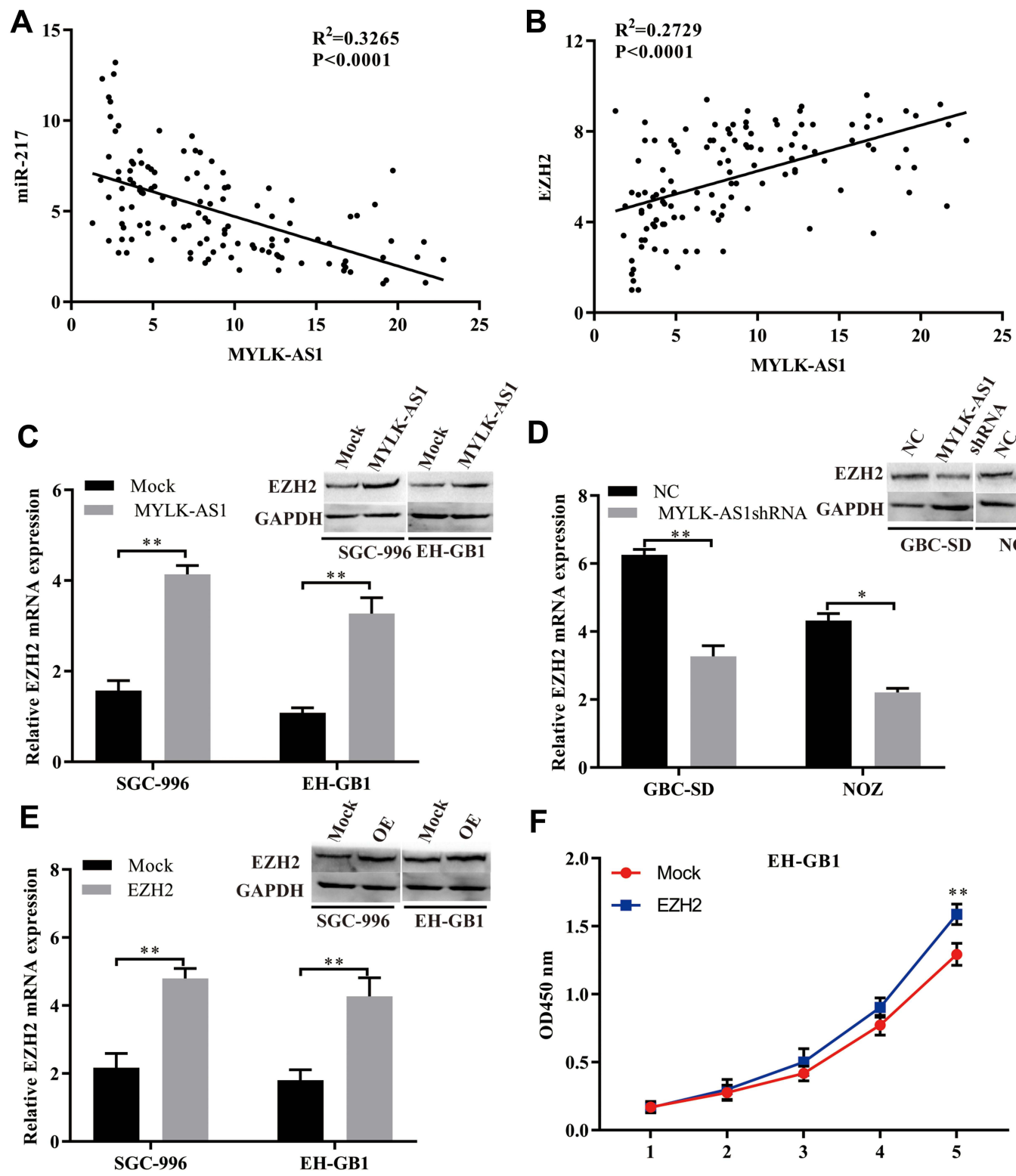

G

D

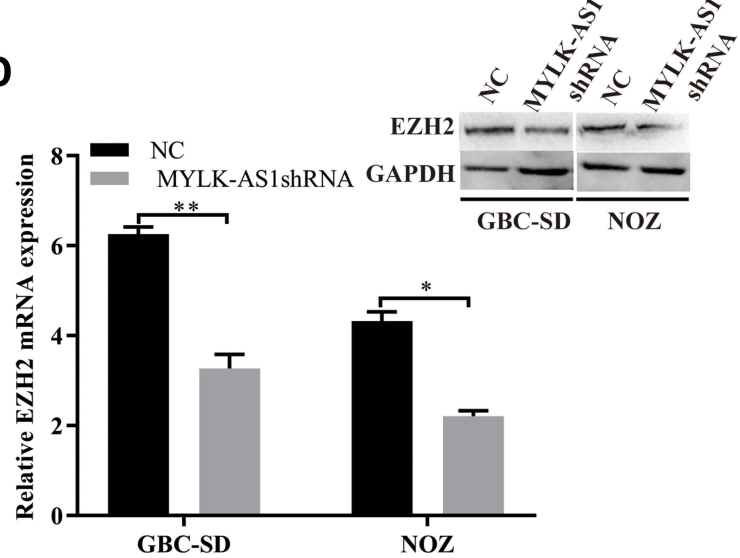

$\mathbf{F}$
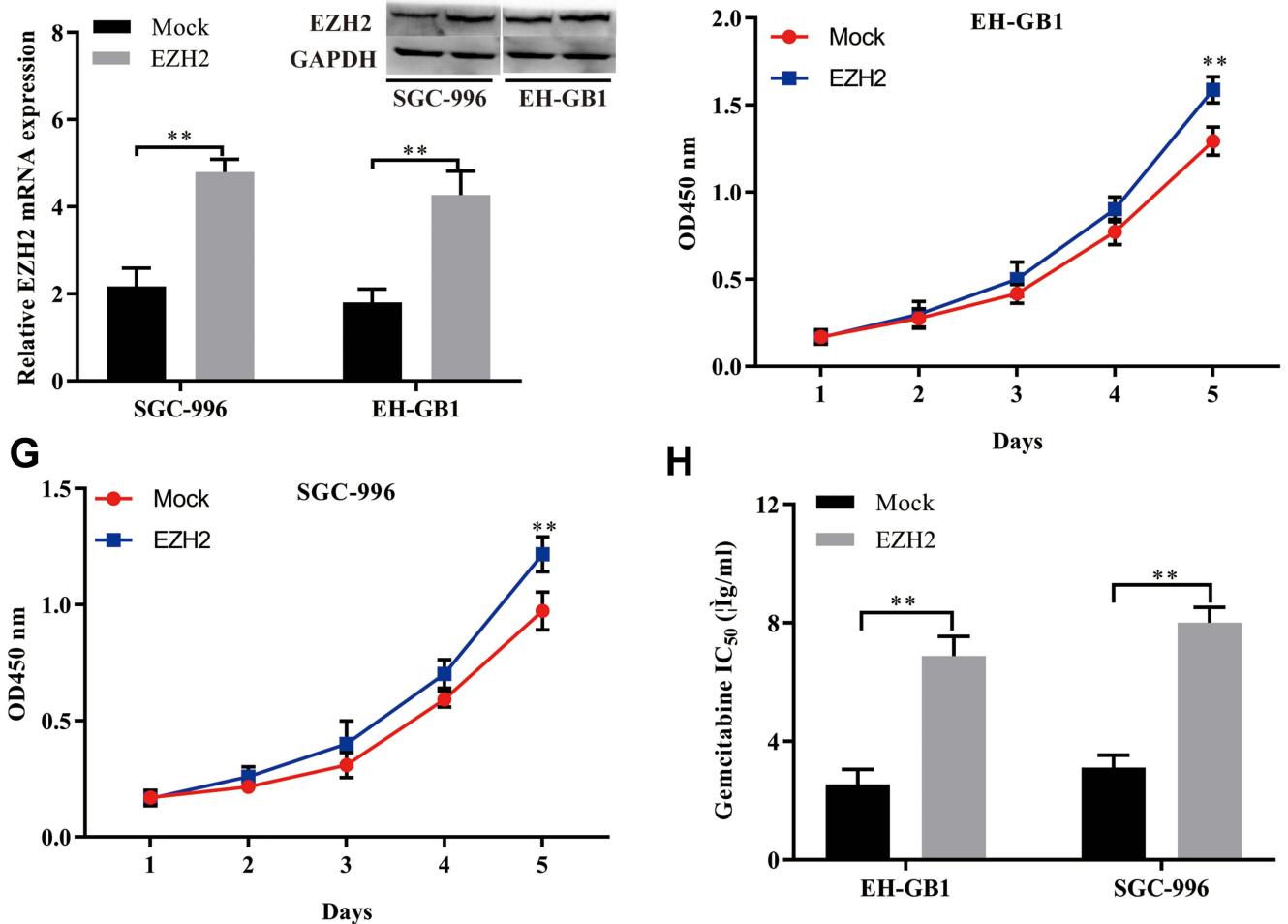

$\mathbf{H}$

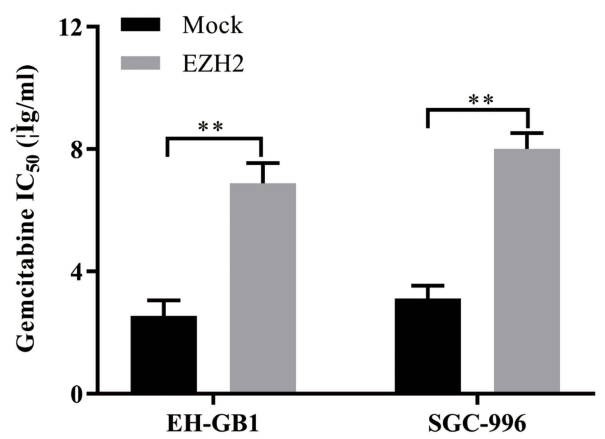

Figure 6 The relationship of MYLK-ASI, miR-217, and EZH2 mRNA in GBC tissues. (A) A negative correlation between MYLK-ASI and miR-217 was observed in GBC tissues. (B) A positive correlation between MYLK-ASI and EZH2 was observed in GBC tissues. (C) The levels of EZH2 were measured by Western blotting and RT-qPCR in GBC cells transfected with MYLK-ASI and the MOCK control. (D) The levels of EZH2 were measured by Western blotting and RT-qPCR in GBC cells transfected with MYLK-ASI shRNA or the negative control. (E) EZH2 expression in GBC cells was modified by plasmid transfection and detected by Western blotting and RT-qPCR. OE: overexpression of EZH2. (F-H) Cell proliferation and drug sensitivity were measured using CCK-8 assays. The data are represented as the mean $\pm S D, n=3$. $* P<0.05$; $* * \mathrm{P}<0.01$. 
a new strategy to overcome chemotherapy resistance in GBC by targeting EZH2.

Taken together, we confirmed that MYLK-AS1 upregulated EZH2 expression through sponge-like binding to miR217 , thus affecting the chemotherapy sensitivity of GBC. Furthermore, forced MYLK-AS1 expression correlated with a poor prognosis and worse clinical characteristics in GBC. In conclusion, our results revealed a concrete molecular mechanism for MYLK-AS1 in GBC, which may serve as a promising biomarker and therapeutic target for GBC.

\section{Data Sharing Statement}

The datasets used during the present study are available from the corresponding author upon reasonable request.

\section{Funding}

This work was supported by a grant from Minhang Hospital Affiliated to Fudan University (2019MHJC10) and the Shanghai Medical Key Subject Construction Project (ZK2019B08).

\section{Disclosure}

The authors state that they have no competing interests.

\section{References}

1. Lee SH, Kim JM, Lee DG, et al. Loss of desmoglein-2 promotes gallbladder carcinoma progression and resistance to EGFR-targeted therapy through Src kinase activation. Cell Death Differ. 2020;28 (3):968-984.

2. Xu S, Zhan M, Jiang C, et al. Genome-wide CRISPR screen identifies ELP5 as a determinant of gemcitabine sensitivity in gallbladder cancer. Nat Commun. 2019;10:5492. doi:10.1038/s41467-019-13420-x

3. Ma L, Bajic VB, Zhang Z. On the classification of long non-coding RNAs. RNA Biol. 2013;10:925-933. doi:10.4161/rna.24604

4. Anastasiadou E, Jacob LS, Slack FJ. Non-coding RNA networks in cancer. Nat Rev Cancer. 2018;18:5-18. doi:10.1038/nrc.2017.99

5. Zhang PF, Wu J, Wu Y, et al. The IncRNA SCARNA2 mediates colorectal cancer chemoresistance through a conserved microRNA-342-3p target sequence. $J$ Cell Physiol. 2019;234: 10157-10165. doi:10.1002/jcp. 27684

6. Luo J, Xiang H. LncRNA MYLK-AS1 acts as an oncogene by epigenetically silencing large tumor suppressor 2 (LATS2) in gastric cancer. Bioengineered. 2021;12:3101-3112. doi:10.1080/21655979.2021.1944019

7. Teng F, Zhang JX, Chang QM, et al. LncRNA MYLK-AS1 facilitates tumor progression and angiogenesis by targeting miR-424-5p/E2F7 axis and activating VEGFR-2 signaling pathway in hepatocellular carcinoma. J Exp Clin Cancer Res. 2020;39:235. doi:10.1186/ s13046-020-01739-z

8. Zhu S, Zhang J, Gao X, et al. Silencing of long noncoding RNA MYLK-AS1 suppresses nephroblastoma via down-regulation of CCNE1 through transcription factor TCF7L2. J Cell Physiol. 2021;236:5757-5770. doi:10.1002/jep.30259

9. Jin L, Cai Q, Wang S, et al. Long noncoding RNA MEG3 regulates LATS2 by promoting the ubiquitination of EZH2 and inhibits proliferation and invasion in gallbladder cancer. Cell Death Dis. 2018;9:1017. doi:10.1038/s41419-018-1064-1
10. Wang SH, Yang $\mathrm{Y}$, Wu XC, et al. Long non-coding RNA MINCR promotes gallbladder cancer progression through stimulating EZH2 expression. Cancer Lett. 2016;380:122-133. doi:10.1016/j.canlet.20 16.06 .019

11. Wu Y, Hu L, Liang Y, et al. Up-regulation of IncRNA CASC9 promotes esophageal squamous cell carcinoma growth by negatively regulating PDCD4 expression through EZH2. Mol Cancer. 2017;16:150. doi:10.1186/s12943-017-0715-7

12. Yiew NKH, Greenway C, Zarzour A, et al. Enhancer of zeste homo$\log 2$ (EZH2) regulates adipocyte lipid metabolism independent of adipogenic differentiation: role of apolipoprotein E. J Biol Chem. 2019;294:8577-8591. doi:10.1074/jbc.RA118.006871

13. Jones BA, Varambally S, Arend RC. Histone methyltransferase EZH2: a therapeutic target for ovarian cancer. Mol Cancer Ther. 2018;17:591-602. doi:10.1158/1535-7163.MCT-17-0437

14. Zhang PF, Gao C, Huang XY, et al. Cancer cell-derived exosomal circUHRF1 induces natural killer cell exhaustion and may cause resistance to anti-PD1 therapy in hepatocellular carcinoma. Mol Cancer. 2020;19:110. doi:10.1186/s12943-020-01222-5

15. Jia B, Xie T, Qiu X, et al. Long noncoding RNA FALEC inhibits proliferation and metastasis of tongue squamous cell carcinoma by epigenetically silencing ECM1 through EZH2. Aging (Albany NY). 2019;11:4990.

16. Zhao Y, Ding L, Wang D, et al. EZH2 cooperates with gain-offunction p53 mutants to promote cancer growth and metastasis. EMBO J. 2019;38:e99599. doi:10.15252/embj.201899599

17. Ramakrishnan S, Granger V, Rak M, et al. Inhibition of EZH2 induces NK cell-mediated differentiation and death in muscle-invasive bladder cancer. Cell Death Differ. 2019;26:2100-2114. doi:10.1038/s41418019-0278-9

18. Xu M, Chen $\mathrm{X}$, Lin $\mathrm{K}$, et al. IncRNA SNHG6 regulates EZH2 expression by sponging $\mathrm{miR}-26 \mathrm{a} / \mathrm{b}$ and $\mathrm{miR}-214$ in colorectal cancer. J Hematol Oncol. 2019;12:3. doi:10.1186/s13045-018-0690-5

19. Jin L, Cai Q, Wang S, Wang S, Wang J, Quan Z. Long noncoding RNA PVT1 promoted gallbladder cancer proliferation by epigenetically suppressing miR-18b-5p via DNA methylation. Cell Death Dis. 2020;11:871. doi:10.1038/s41419-020-03080-x

20. Yang G, Wang J, Huang B. Loss of miR-217 promotes osteosarcoma cell proliferation through targeting SETD8. Pharmazie. 2018;73:711-714.

21. Gao Y, Wang B, Luo H, Zhang Q, Xu M. miR-217 represses TGF-beta1-induced airway smooth muscle cell proliferation and migration through targeting ZEB1. Biomed Pharmacother. 2018;108:27-35. doi:10.1016/j.biopha.2018.09.030

22. Pan B, Yang J, Wang X, Xu K, Ikezoe T. miR-217 sensitizes chronic myelogenous leukemia cells to tyrosine kinase inhibitors by targeting pro-oncogenic anterior gradient 2. Exp Hematol. 2018;68:80-88e2. doi:10.1016/j.exphem.2018.09.001

23. Yan J, Wu G, Chen J, Xiong L, Chen G, Li P. Downregulated miR-217 expression predicts a poor outcome in acute myeloid leukemia. Cancer Biomark. 2018;22:73-78. doi:10.3233/CBM-170936

24. Flum M, Kleemann M, Schneider H, et al. miR-217-5p induces apoptosis by directly targeting PRKCI, BAG3, ITGAV and MAPK1 in colorectal cancer cells. J Cell Commun Signal. 2018;12:451-466. doi:10.1007/s12079-017-0410-x

25. Dong J, Wang M, Ni D, et al. MicroRNA-217 functions as a tumor suppressor in cervical cancer cells through targeting Rho-associated protein kinase 1. Oncol Lett. 2018;16:5535-5542.

26. Heckmann D, Maier $\mathrm{P}$, Laufs $\mathrm{S}$, et al. The disparate twins: a comparative study of CXCR4 and CXCR7 in SDF-1alphainduced gene expression, invasion and chemosensitivity of colon cancer. Clin Cancer Res. 2014;20:604-616. doi:10.1158/1078-0432. CCR-13-0582

27. Wang H, Qin R, Guan A, et al. HOTAIR enhanced paclitaxel and doxorubicin resistance in gastric cancer cells partly through inhibiting miR-217 expression. J Cell Biochem. 2018;119:7226-7234. doi:10. $1002 /$ jcb. 26901 
28. Guo J, Feng Z, Huang Z, Wang H, Lu W. MicroRNA-217 functions as a tumour suppressor gene and correlates with cell resistance to cisplatin in lung cancer. Mol Cells. 2014;37:664-671. doi:10.14348/ molcells.2014.0121

29. Margueron R, Reinberg D. The polycomb complex PRC2 and its mark in life. Nature. 2011;469:343-349. doi:10.1038/nature09784

30. Qiu X, Wang W, Li B, et al. Targeting Ezh2 could overcome docetaxel resistance in prostate cancer cells. BMC Cancer. 2019;19:27. doi:10.1186/s12885-018-5228-2
31. Wu Y, Zhang Z, Cenciarini ME, et al. Tamoxifen resistance in breast cancer is regulated by the EZH2-ERalpha-GREB1 transcriptional axis. Cancer Res. 2018;78:671-684. doi:10.1158/0008-5472.CAN$17-1327$

\section{Publish your work in this journal}

Cancer Management and Research is an international, peer-reviewed open access journal focusing on cancer research and the optimal use of preventative and integrated treatment interventions to achieve improved outcomes, enhanced survival and quality of life for the cancer patient.
The manuscript management system is completely online and includes a very quick and fair peer-review system, which is all easy to use. Visit http://www.dovepress.com/testimonials.php to read real quotes from published authors. 